\title{
ON THE ANALOGY BETWEEN SELF-GRAVITATING BROWNIAN PARTICLES AND BACTERIAL POPULATIONS
}

\author{
PIERRE-HENRI CHAVANIS \\ Laboratoire de Physique Théorique, Université Paul Sabatier \\ 118 route de Narbonne, 31062 Toulouse Cedex 4, France \\ E-mail: chavanis@irsamc.ups-tlse.fr \\ MAGALI RIBOT \\ MAPLY, Université Claude Bernard \\ Bâtiment 101, 69622 Villeurbanne Cedex, France \\ E-mail: ribot@maply.univ-lyon1.fr \\ CAROLE ROSIER \\ LAPCS, Université Claude Bernard \\ 50 avenue Tony Garnier, 69366 Lyon, France \\ E-mail: rosier@maply.univ-lyon1.fr \\ CLÉMENT SIRE \\ Laboratoire de Physique Théorique, Université Paul Sabatier \\ 118 route de Narbonne, 31062 Toulouse Cedex 4, France \\ E-mail: clement@irsamc.ups-tlse.fr
}

\begin{abstract}
We develop the analogy between self-gravitating Brownian particles and bacterial populations. In the high friction limit, the self-gravitating Brownian gas is described by the Smoluchowski-Poisson system. These equations can develop a self-similar collapse leading to a finite time singularity. Coincidentally, the Smoluchowski-Poisson system corresponds to a simplified version of the Keller-Segel model of bacterial populations. In this biological context, it describes the chemotactic aggregation of the bacterial colonies. We extend these classical models by introducing a small-scale regularization. In the gravitational context, we consider a gas of self-gravitating Brownian fermions and in the biological context we consider finite size effects. In that case, the collapse stops when the system feels the influence of the small-scale regularization. A phenomenon of "explosion", reverse to the collapse, is also possible.
\end{abstract}

2000 Mathematics Subject Classification: 92-xx, 85-xx, 35-xx, 82-xx.

The paper is in final form and no version of it will be published elsewhere. 
1. Introduction. Self-gravitating systems such as globular clusters and elliptical galaxies form a Hamiltonian system of particles in interaction that can be supposed isolated in a first approximation [1]. Since energy is conserved, the proper statistical description of stellar systems is the microcanonical ensemble [2]. The dynamical evolution of elliptical galaxies is governed by the Vlasov-Poisson system which corresponds to a collisionless regime. On the other hand, the kinetic theory of stars in globular clusters is based on the Landau equation (or the orbit averaged Fokker-Planck equation) which describes a collisional evolution. These equations conserve mass and energy. Furthermore, the Landau equation increases the Boltzmann entropy (H-theorem) due to stellar encounters. These equations have been studied for a long time in the astrophysical literature and a relatively good physical understanding has now been achieved. In particular, globular clusters can experience core collapse related to the "gravothermal catastrophe" [3].

For systems with long-range interactions, statistical ensembles are not equivalent. Therefore, it is of conceptual interest to compare the microcanonical evolution of stellar systems to a canonical model. This can be achieved by considering a gas of selfgravitating Brownian particles submitted to a friction with an inert gas and a stochastic force, in addition to self-gravity [4]. This system has a rigorous canonical structure. In the mean-field approximation, the self-gravitating Brownian gas model is described by the Kramers-Poisson system. In a strong friction limit, or for large times, it reduces to the Smoluchowski-Poisson system. These equations conserve mass and decrease the Boltzmann free energy. They possess a rich physical and mathematical structure and can lead to a situation of "isothermal collapse" [5], which is the canonical version of the "gravothermal catastrophe". These equations have not been considered by astrophysicists because the canonical ensemble is not the correct description of stellar systems and usual astrophysical bodies do not experience a friction with a gas (except dust particles in the solar nebula [6]). Yet, it is clear that the self-gravitating Brownian gas model is of considerable conceptual interest to understand the strange thermodynamics of systems with long-range interactions and the inequivalence of statistical ensembles.

In addition, it turns out that the same type of equations occur in biology in relation with the chemotactic aggregation of bacterial populations [7]. A general model of chemotactic aggregation has been proposed by Keller \& Segel [8] in the form of two coupled differential equations. In some approximation [9], this model reduces to the SmoluchowskiPoisson system, exactly like for self-gravitating Brownian particles. Therefore, there exists an isomorphism between self-gravitating Brownian particles and bacterial colonies. In this paper, we shall develop this analogy in detail. We shall also propose a modification of the "standard model" by introducing a small-scale regularization. In the gravitational context, we shall invoke Pauli's exclusion principle and consider a gas of self-gravitating Brownian fermions. In the biological context, we shall heuristically account for finite size effects by considering a lattice model. In that case, the collapse stops when the system feels the small-scale regularization. An explosion phenomenon, reverse to the collapse, is also possible. Finally, we shall discuss the difference between elliptical and parabolic models of bacterial populations and gravitational systems. We shall also show that vortices in two-dimensional turbulence exhibit features similar to stars and bacteries. 
2. The Hamiltonian $N$-stars problem. Consider a system of $N$ stars in gravitational interaction. We assume that the system is isolated so that it conserves mass and energy. The equations of motion can be cast in a Hamiltonian form

$$
m \frac{d \mathbf{r}_{i}}{d t}=\frac{\partial H}{\partial \mathbf{v}_{i}}, \quad m \frac{d \mathbf{v}_{i}}{d t}=-\frac{\partial H}{\partial \mathbf{r}_{i}},
$$

where $H$ is the Hamiltonian

$$
H=\frac{1}{2} \sum_{i=1}^{N} m v_{i}^{2}-\sum_{i<j} \frac{G m^{2}}{\left|\mathbf{r}_{i}-\mathbf{r}_{j}\right|} .
$$

This $N$-body problem is the correct starting point in the description of globular clusters and elliptical galaxies [1]. Since the system is isolated, the relevant statistical ensemble is the microcanonical ensemble [2]. The statistical mechanics of self-gravitating systems enclosed within a spherical box of radius $R$ was initiated by Antonov [10] and LyndenBell \& Wood [3]. They found that for $\Lambda \equiv-E R / G M^{2} \geq 0.335$, corresponding to small energies, there is no maximum entropy state so that the system must collapse (see Fig. 1). This is the so-called "gravothermal catastrophe".

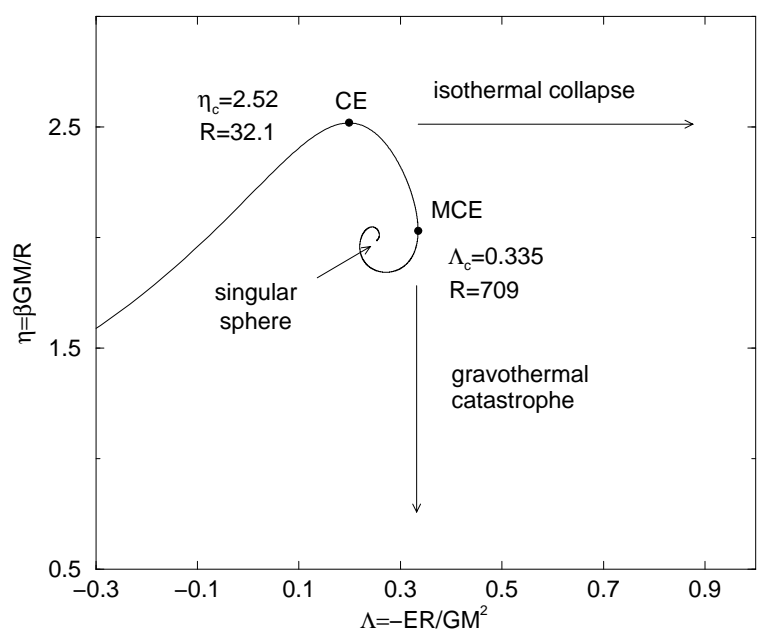

Fig. 1. Caloric curve for self-gravitating systems exhibiting a "gravothermal catastrophe" in the microcanonical ensemble and an "isothermal collapse" in the canonical ensemble.

In the case of elliptical galaxies, encounters between stars are completely negligible for the timescales of interest and the dynamics is described by the Vlasov-Poisson system

$$
\begin{gathered}
\frac{\partial f}{\partial t}+\mathbf{v} \cdot \frac{\partial f}{\partial \mathbf{r}}+\mathbf{F} \cdot \frac{\partial f}{\partial \mathbf{v}}=0, \\
\Delta \Phi=4 \pi G \int f d^{3} \mathbf{v},
\end{gathered}
$$

where $f(\mathbf{r}, \mathbf{v}, t)$ is the distribution function, $\mathbf{F}(\mathbf{r}, t)=-\nabla \Phi$ is the gravitational force and $\Phi(\mathbf{r}, t)$ is the gravitational potential. By contrast, for globular clusters, stellar encounters must be taken into account. In that case, the dynamics is described by the Landau 
equation

$$
\frac{\partial f}{\partial t}+\mathbf{v} \cdot \frac{\partial f}{\partial \mathbf{r}}+\mathbf{F} \cdot \frac{\partial f}{\partial \mathbf{v}}=\frac{\partial}{\partial v^{\mu}} \int d^{3} \mathbf{v}_{1} K^{\mu \nu}\left\{f_{1} \frac{\partial f}{\partial v^{\nu}}-f \frac{\partial f_{1}}{\partial v_{1}^{\nu}}\right\}
$$

where $K^{\mu \nu}$ is the tensor

$$
K^{\mu \nu}=\frac{A}{u}\left(\delta^{\mu \nu}-\frac{u^{\mu} u^{\nu}}{u^{2}}\right),
$$

where $f_{1}=f\left(\mathbf{r}, \mathbf{v}_{1}, t\right), \mathbf{u}=\mathbf{v}_{1}-\mathbf{v}$ is the relative velocity and $A=2 \pi G^{2} m \ln \left(L_{\max } / L_{\min }\right)$ is a constant $\left(L_{\max }\right.$ and $L_{\min }$ are appropriate lengthscales). This equation conserves mass $M=\int \rho d^{3} \mathbf{r}$ and energy $E=\int f \frac{v^{2}}{2} d^{3} \mathbf{r} d^{3} \mathbf{v}+\frac{1}{2} \int \rho \Phi d^{3} \mathbf{r}$ and satisfies an H-theorem $(\dot{S} \geq 0)$ for the Boltzmann entropy

$$
S[f]=-\int f \ln f d^{3} \mathbf{r} d^{3} \mathbf{v}
$$

Therefore, due to the development of encounters between stars, the system is expected to relax towards a statistical equilibrium state described by the Maxwell-Boltzmann distribution

$$
f=A e^{-\beta\left(\frac{v^{2}}{2}+\Phi\right)},
$$

which maximizes the Boltzmann entropy at fixed mass and energy (microcanonical description). In fact, this is the case only if the energy is sufficiently high. Below the Antonov critical energy, there is no maximum entropy state and the system undergoes core collapse. This is a manifestation of the "gravothermal catastrophe". Dynamical models show that the collapse is self-similar and leads to a finite time singularity (the central density becomes infinite in a finite time). By solving the orbit averaged Fokker-Planck equation numerically, Cohn [11] finds that the density profile behaves as $\rho \sim r^{-\alpha}$ with $\alpha=2.23$ at large distances. Alternatively, Lancellotti \& Kiessling [12] consider the full LandauPoisson system and argue that $\alpha=3$.

3. The self-gravitating Brownian gas. The Hamiltonian equations (1)-(2) describe an isolated system for which energy is conserved. It can be of interest to study in parallel a model that is stochastically forced by an external medium. We thus introduce a system of $N$ Brownian particles in gravitational interaction described by the Langevin equations

$$
\frac{d \mathbf{r}_{i}}{d t}=\mathbf{v}_{i}, \quad \frac{d \mathbf{v}_{i}}{d t}=-\xi \mathbf{v}_{i}-\nabla_{i} U\left(\mathbf{r}_{1}, \ldots, \mathbf{r}_{N}\right)+\sqrt{2 D} \mathbf{R}_{i}(t)
$$

where $-\xi \mathbf{v}_{i}$ is a friction force and $\mathbf{R}_{i}(t)$ is a white noise satisfying $\left\langle\mathbf{R}_{i}(t)\right\rangle=\mathbf{0}$ and $\left\langle R_{a, i}(t) R_{b, j}\left(t^{\prime}\right)\right\rangle=\delta_{i j} \delta_{a b} \delta\left(t-t^{\prime}\right)$, where $a, b=1,2,3$ refer to the coordinates of space and $i, j=1, \ldots, N$ to the particles. The particles interact via the gravitational potential $U\left(\mathbf{r}_{1}, \ldots, \mathbf{r}_{N}\right)=\sum_{i<j} u\left(\mathbf{r}_{i}-\mathbf{r}_{j}\right)$ where $u\left(\mathbf{r}_{i}-\mathbf{r}_{j}\right)=-G /\left|\mathbf{r}_{i}-\mathbf{r}_{j}\right|$. We define the inverse temperature $\beta=1 / T$ through the Einstein relation $\xi=D \beta$. The self-gravitating Brownian gas model has a rigorous canonical structure (see Appendices A and B) where the temperature $T$ measures the strength of the stochastic force. The stochastic process (9) defines a model of gravitational dynamics which extends the classical EinsteinSmoluchowski Brownian model [13] to the case of stochastic particles in interaction. In this context, the friction is due to the presence of an inert gas and the stochastic force is due to classical Brownian motion, turbulence or any other stochastic effect. This model 
can also be viewed as a generalization of the Chandrasekhar [14] stochastic model which describes the evolution of a single test particle in a stellar cluster at statistical equilibrium (thermal bath approximation). In that context, the diffusion and the friction model stellar encounters. More generally, the friction and the noise can mimick the overall influence of an external medium (not represented) with which the particles interact.

Starting from the $N$-body Fokker-Planck equation and implementing a mean-field approximation which is valid in a proper thermodynamic limit $N \rightarrow+\infty$ with $\eta=$ $\beta G M / R$ fixed, we show in Appendix B that the distribution function $f(\mathbf{r}, \mathbf{v}, t)$ satisfies the Kramers-Poisson system

$$
\begin{gathered}
\frac{\partial f}{\partial t}+\mathbf{v} \cdot \frac{\partial f}{\partial \mathbf{r}}+\mathbf{F} \cdot \frac{\partial f}{\partial \mathbf{v}}=\frac{\partial}{\partial \mathbf{v}} \cdot\left\{D\left[\frac{\partial f}{\partial \mathbf{v}}+\beta f \mathbf{v}\right]\right\} \\
\Delta \Phi=4 \pi G \int f d^{3} \mathbf{v} .
\end{gathered}
$$

The Kramers-Poisson system decreases the Boltzmann free energy

$$
F[f]=E-T S=\int f \frac{v^{2}}{2} d^{3} \mathbf{r} d^{3} \mathbf{v}+\frac{1}{2} \int \rho \Phi d^{3} \mathbf{r}+T \int f \ln f d^{3} \mathbf{r} d^{3} \mathbf{v} .
$$

Therefore, the system is expected to relax towards a statistical equilibrium state described by the Maxwell-Boltzmann distribution

$$
f=A e^{-\beta\left(\frac{v^{2}}{2}+\Phi\right)}
$$

which minimizes the Boltzmann free energy at fixed mass and temperature (canonical description). In fact, this is the case only if the temperature is sufficiently high. Below a critical temperature [5], the free energy has no minimum and the system undergoes an "isothermal collapse" (see Fig. 1). We note that the equilibrium states of stellar systems (e.g., globular clusters) and self-gravitating Brownian particles are both given by the isothermal distribution (8) or (13). However, the stability limits are different in each ensemble because the caloric curve $\beta(E)$ presents turning points (see Fig. 1). The stability of stellar systems and self-gravitating Brownian particles differs in the region of ensemble inequivalence where the specific heat $C=d E / d T$ is negative [5].

In the high friction limit $\xi \rightarrow+\infty$, or equivalently for large times $t \gg \xi^{-1}$, we can neglect the inertia of the particles so that the Langevin equations (9) take the form

$$
\xi \frac{d \mathbf{r}_{i}}{d t}=-\nabla_{i} U\left(\mathbf{r}_{1}, \ldots, \mathbf{r}_{N}\right)+\sqrt{2 D} \mathbf{R}_{i}(t)
$$

Again using a mean-field approximation (see Appendix B), we can show that the density $\rho(\mathbf{r}, t)$ satisfies the Smoluchowski-Poisson system

$$
\begin{gathered}
\frac{\partial \rho}{\partial t}=\nabla \cdot\left[\frac{1}{\xi}(T \nabla \rho+\rho \nabla \Phi)\right], \\
\Delta \Phi=4 \pi G \rho .
\end{gathered}
$$

The Smoluchowski equation (15) can also be deduced from the Kramers equation (10) by using a method of moments [15] or a Chapman-Enskog expansion [16]. We can show that it decreases the Boltzmann free energy 


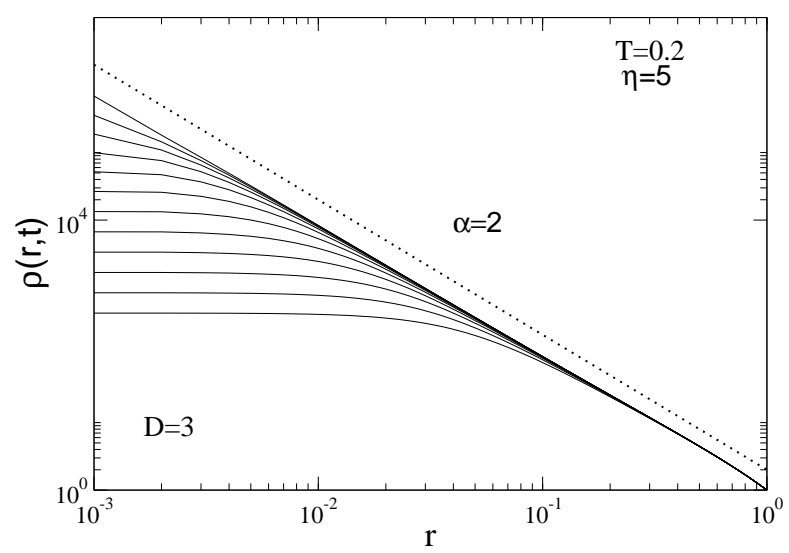

Fig. 2. Isothermal collapse in $D=3$. For $\eta=\beta G M / R>2.52$, the system undergoes a finite time singularity leading to a $\rho \sim r^{-2}$ density profile $[4,19]$. A Dirac peak is formed in the post-collapse regime [21].

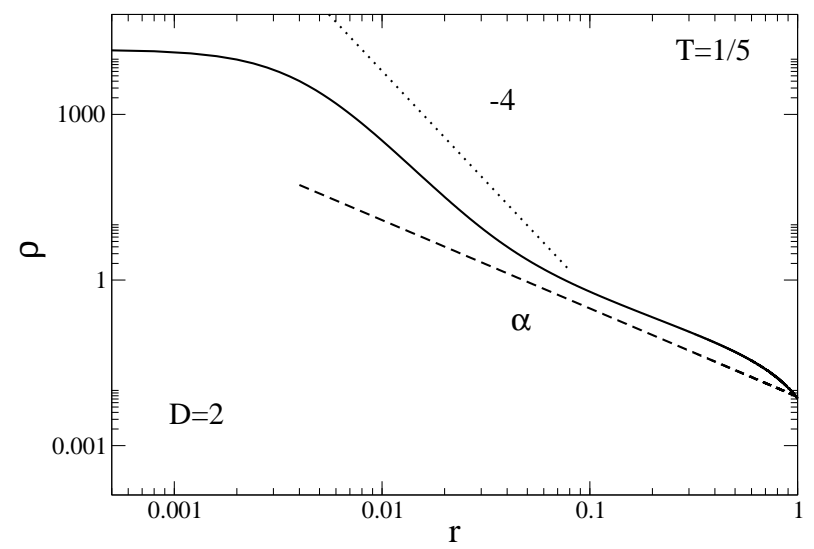

Fig. 3. Isothermal collapse in $D=2$. For $\eta=\beta G M>4$, the system develops a Dirac peak surrounded by a halo behaving as $\rho \sim r^{-\alpha}$. For $t \rightarrow t_{\text {coll }}, \alpha=2$ but the convergence to this asymptotic value is extremely slow so that an effective exponent $\alpha \simeq 1.3$ is observed for the times achieved in our simulations [19].

$$
F[\rho]=T \int \rho \ln \rho d^{3} \mathbf{r}+\frac{1}{2} \int \rho \Phi d^{3} \mathbf{r},
$$

obtained from Eq. (12) by using the fact that the distribution function is close to the Maxwellian distribution

$$
f=\left(\frac{\beta}{2 \pi}\right)^{3 / 2} \rho(\mathbf{r}, t) e^{-\beta \frac{v^{2}}{2}},
$$

in the high friction limit $[15,16]$. The equilibrium states of the Smoluchowski-Poisson system (15)-(16) are given by the Boltzmann distribution

$$
\rho=A^{\prime} e^{-\beta \Phi} \text {. }
$$


In the gravitational context, the Kramers-Poisson system and the SmoluchowskiPoisson system have been introduced by Wolansky [17], Chavanis, Sommeria \& Robert [18] and Chavanis, Rosier \& Sire [4] with different motivations. A physical description of the spherically symmetric solutions of the Smoluchowski-Poisson system has been given in $[4,19,20,21,22]$ in various dimensions of space (including the critical dimension $D=2$ ) and for both pre-collapse and post-collapse regimes. For sufficiently large mass $M$ or sufficiently low temperature $T$, the Smoluchowski-Poisson system displays a self-similar collapse leading to a finite time singularity in $D>2$ (see Fig. 2). The density profile behaves as $\rho \sim r^{-\alpha}$ with $\alpha=2$ at the collapse time $[4,19]$. Then, the evolution continues and a Dirac peak ("black hole") is finally formed in the post-collapse regime [21]. This is consistent with predictions of statistical mechanics in the canonical ensemble [23, 5]. In $D=2$, the evolution is more complex [19] and creates a Dirac peak containing a fraction $T / T_{c}$ of the total mass, surrounded by a $\rho \sim r^{-\alpha}$ halo with an effective scaling exponent converging very slowly to $\alpha=2$ (see Fig. 3). A review of these results is given in [24].

4. Self-gravitating Brownian fermions. As discussed previously, self-gravitating classical particles have the tendency to develop finite time singularities. In an attempt to regularize the problem at high densities, and avoid unphysical infinities, we can invoke quantum mechanics and use Pauli's exclusion principle. Thus, we shall consider a gas of self-gravitating Brownian fermions as a regularized model of gravitational dynamics. The equilibrium states of self-gravitating fermions and the description of phase transitions in the self-gravitating Fermi gas (in both microcanonical and canonical ensembles) have been investigated by Chavanis [25, 26]. The system of self-gravitating Brownian fermions [27] can be used as a simple model to study these phase transitions dynamically in the canonical ensemble.

A generalization of the Kramers equation (10) taking into account the Pauli exclusion principle is given by [15]:

$$
\frac{\partial f}{\partial t}+\mathbf{v} \cdot \frac{\partial f}{\partial \mathbf{r}}+\mathbf{F} \cdot \frac{\partial f}{\partial \mathbf{v}}=\frac{\partial}{\partial \mathbf{v}} \cdot\left\{D\left[\frac{\partial f}{\partial \mathbf{v}}+\beta f\left(1-f / \eta_{0}\right) \mathbf{v}\right]\right\}
$$

This equation respects the constraint $f \leq \eta_{0} \equiv m^{4} / h^{3}$ ( $h$ is Planck constant) at all times and decreases the Fermi-Dirac free energy

$$
F=\int f \frac{v^{2}}{2} d^{3} \mathbf{r} d^{3} \mathbf{v}+\frac{1}{2} \int \rho \Phi d^{3} \mathbf{r}+T \int\left\{f \ln f+\left(\eta_{0}-f\right) \ln \left(\eta_{0}-f\right)\right\} d^{3} \mathbf{r} d^{3} \mathbf{v}
$$

The equilibrium states are given by the Fermi-Dirac distribution function

$$
f=\frac{\eta_{0}}{1+\lambda e^{\beta\left(\frac{v^{2}}{2}+\Phi\right)}}
$$

which minimizes the free energy (21) at fixed mass and temperature.

Now, considering the high friction limit $[15,16]$, we can derive a generalized Smoluchowski equation of the form

$$
\frac{\partial \rho}{\partial t}=\nabla \cdot\left[\frac{1}{\xi}(\nabla p+\rho \nabla \Phi)\right]
$$


where $p(\rho)$ is the local equation of state of the Fermi gas

$$
\rho=\frac{4 \pi \sqrt{2} \eta_{0}}{\beta^{3 / 2}} I_{\frac{1}{2}}\left(\lambda^{\prime}\right), \quad p=\frac{8 \pi \sqrt{2} \eta_{0}}{3 \beta^{5 / 2}} I_{\frac{3}{2}}\left(\lambda^{\prime}\right),
$$

and

$$
I_{n}(t)=\int_{0}^{+\infty} \frac{x^{n}}{1+t e^{x}} d x
$$

is the Fermi integral. The fermionic Smoluchowski-Poisson system decreases the free energy

$$
F[\rho]=\int \rho \int_{0}^{\rho} \frac{p\left(\rho^{\prime}\right)}{\rho^{\prime 2}} d \rho^{\prime} d^{D} \mathbf{r}+\frac{1}{2} \int \rho \Phi d^{D} \mathbf{r},
$$

obtained from Eq. (21) by using the fact that $f(\mathbf{r}, \mathbf{v}, t)$ is close to the Fermi distribution in the high friction limit [16]. Furthermore, the equilibrium states are obtained by substituting the relation

$$
\rho=\frac{4 \pi \sqrt{2} \eta_{0}}{\beta^{3 / 2}} I_{\frac{1}{2}}\left(\lambda e^{\beta \Phi}\right),
$$

in the Poisson equation (16) and solving for the gravitational potential $\Phi$ [25].

The fermionic Smoluchowski-Poisson system has been studied mathematically by Biler et al. [28]. Numerical simulations have been conducted in parallel in [27]. In particular, these simulations reveal an interesting hysteretic cycle (see Fig. 4) discussed by Chavanis \& Rieutord [29]. Below a critical temperature $T_{c}$, coinciding with the Jeans instability criterion $[4,5]$, the system collapses under its own gravity as in Fig. 2. However, for self-

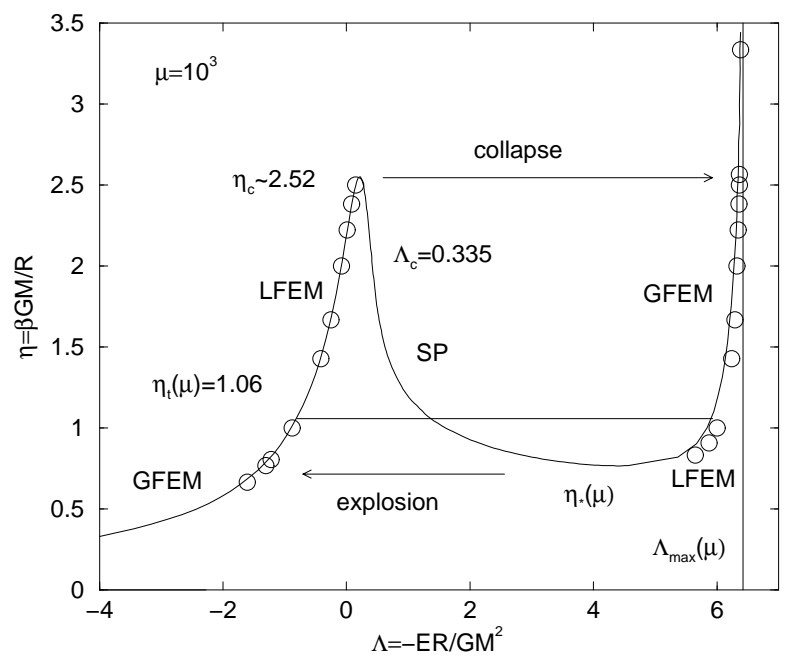

Fig. 4. Hysteretic cycle in the canonical ensemble for self-gravitating fermions. The system undergoes a "collapse" at $T_{c}$ and an "explosion" at $T_{*}$. The temperatures of collapse and explosion differ due to the existence of long-lived metastable states (local minima of free energy) [29]. The circles correspond to the results of numerical simulations of the fermionic Smoluchowski-Poisson system [27]. 


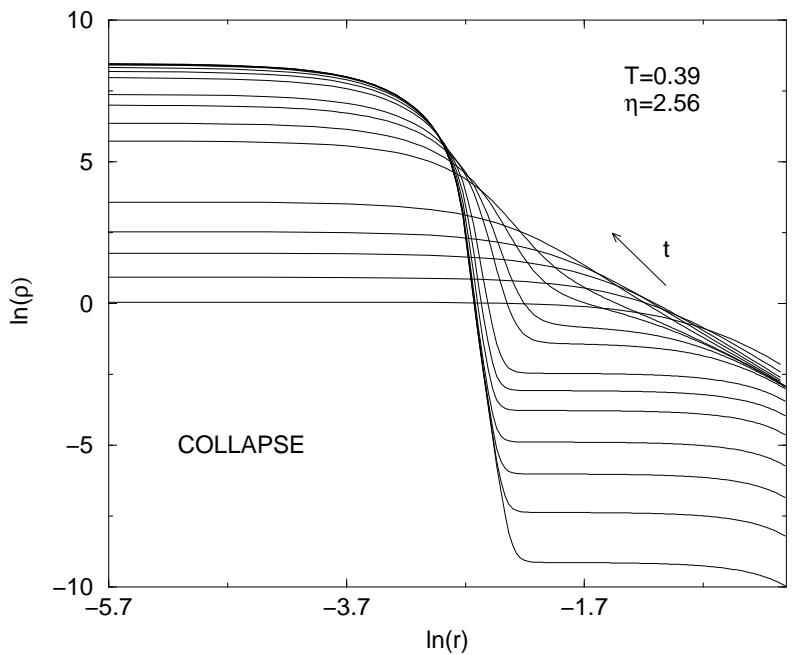

Fig. 5. Collapse of self-gravitating Brownian fermions for $T<T_{c}$. The system finally forms a "fermion ball" (similar to a white dwarf star) surrounded by an isothermal halo (like a vapor). This is a global minimum of free energy in the canonical ensemble.

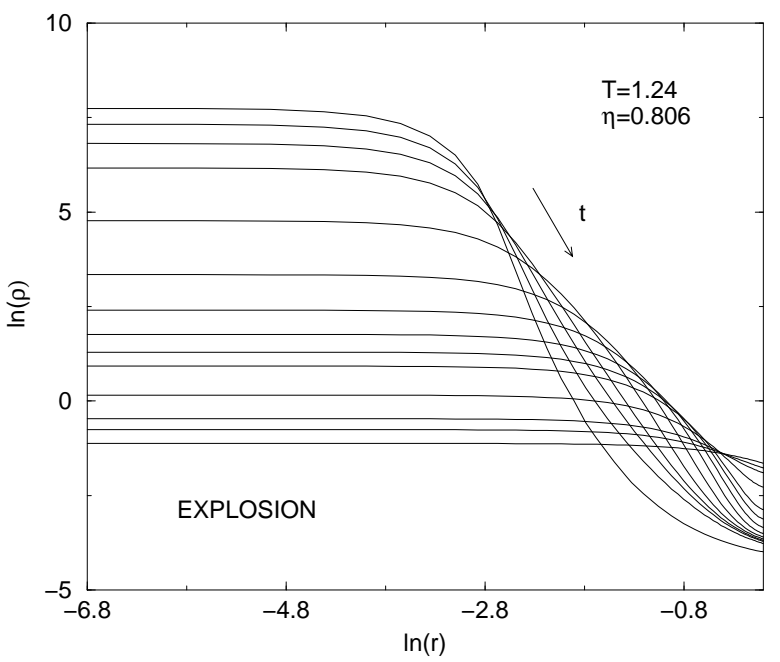

Fig. 6. Explosion of self-gravitating Brownian fermions for $T>T_{*}$. When diffusion prevails over gravity, the condensed object expands and the system returns to the gaseous phase.

gravitating fermions, the collapse stops when the core becomes degenerate (in the sense of the Fermi-Dirac statistics). The resulting condensed object has the same structure as a cold white dwarf star (or a "fermion ball") in which gravity is balanced by quantum pressure (see Fig. 5). If now temperature is increased, the system remains in the condensed phase until another temperature $T_{*}>T_{c}$ (see Fig. 4) at which it undergoes an explosion (reverse to the collapse) and returns to the gaseous phase (see Fig. 6). 
5. Chemotactic aggregation. We shall now point out some analogies between a gas of self-gravitating Brownian particles and the chemotaxis of bacterial populations [7]. The name chemotaxis refers to the motion of organisms (amoeba) induced by chemical signals (acrasin). In some cases, the biological organisms secrete a substance that has an attractive effect on the organisms themselves. Therefore, in addition to their diffusive motion, they move systematically along the gradient of concentration of the chemical they secrete (chemotactic flux). When attraction prevails over diffusion, the chemotaxis can trigger a self-accelerating process until a point at which aggregation takes place. This is the case for the slime mold Dictyostelium Discoideum and for the bacteria Escherichia coli.

A model of slime mold aggregation has been introduced by Keller \& Segel [8] in the form of two PDE's:

$$
\begin{gathered}
\frac{\partial \rho}{\partial t}=\nabla \cdot\left(D_{2} \nabla \rho\right)-\nabla \cdot\left(D_{1} \nabla c\right), \\
\frac{\partial c}{\partial t}=-k(c) c+f(c) \rho+D_{c} \Delta c .
\end{gathered}
$$

In these equations $\rho(\mathbf{r}, t)$ is the concentration of amoebae and $c(\mathbf{r}, t)$ is the concentration of acrasin. Acrasin is produced by the amoebae at a rate $f(c)$. It can also be degraded at a rate $k(c)$. Acrasin diffuse according to Fick's law with a diffusion coefficient $D_{c}$. Amoebae concentration changes as a result of an oriented chemotactic motion in the direction of a positive gradient of acrasin and a random motion analogous to diffusion. In Eq. $(28), D_{2}(\rho, c)$ is the diffusion coefficient of the amoebae and $D_{1}(\rho, c)$ is a measure of the strength of the influence of the acrasin gradient on the flow of amoebae. This chemotactic drift is the fundamental process in the problem.

A first simplification of the Keller-Segel model is provided by the system of equations

$$
\begin{gathered}
\frac{\partial \rho}{\partial t}=D \Delta \rho-\chi \nabla \cdot(\rho \nabla c) \\
\frac{\partial c}{\partial t}=D^{\prime} \Delta c+a \rho-b c
\end{gathered}
$$

where the parameters are positive constants. An additional simplification, introduced by Jäger \& Luckhaus [9], consists in ignoring the temporal derivative in Eq. (31). This is valid in the case where the diffusion coefficient $D^{\prime}$ is large. Taking also $b=0$, we obtain

$$
\begin{gathered}
\frac{\partial \rho}{\partial t}=D \Delta \rho-\chi \nabla \cdot(\rho \nabla c), \\
\Delta c=-\lambda \rho,
\end{gathered}
$$

where $\lambda=a / D^{\prime}$. Clearly, these equations are isomorphic to the Smoluchowski-Poisson system (15)-(16) describing self-gravitating Brownian particles in a high friction limit. In particular, the chemotactic flux plays the same role as the gravitational drift in the overdamped limit of the Brownian model. We have the correspondence $\Phi \leftrightarrow-\frac{4 \pi G}{\lambda} c, \beta \leftrightarrow$ $\frac{\lambda \chi}{4 \pi G D}$ and $\xi \leftrightarrow \frac{4 \pi G}{\lambda \chi}$. Through this analogy, we can develop an effective thermodynamical formalism to investigate the chemotactic problem [15, 30]. In particular, Eq. (32) is similar to a Fokker-Planck equation and its stationary solutions are similar to the Boltzmann 
distribution

$$
\rho=A e^{\frac{\chi}{D} c}
$$

which maximizes a Lyapunov functional similar to the Boltzmann free energy.

6. A generalized chemotactic model. The Keller-Segel model ignores clumping and sticking effects. However, at the late stages of the blow-up, when the density of amoebae has reached high values, finite size effects and stickiness must clearly be taken into account. As a first step, we propose to replace the classical equation (32) by an equation of the form

$$
\frac{\partial \rho}{\partial t}=D \Delta \rho-\chi \nabla \cdot\left(\rho\left(1-\rho / \sigma_{0}\right) \nabla c\right)
$$

which enforces a limitation $\rho \leq \sigma_{0}$ on the maximum concentration of bacteria in physical space [31]. This equation increases the Lyapunov functional

$$
J[\rho]=-\int\left[\rho \ln \rho+\left(\sigma_{0}-\rho\right) \ln \left(\sigma_{0}-\rho\right)\right] d^{3} \mathbf{r}+\frac{\chi}{2 D} \int \rho c d^{3} \mathbf{r} .
$$

In the thermodynamical analogy mentioned above, this functional can be interpreted as a free energy $J=S-\beta E$ associated with a Fermi-Dirac entropy in physical space $[15,30]$. This form of entropy can be obtained by introducing a lattice model preventing two particles to be on the same site. The lattice creates an exclusion principe in physical space similar to the Pauli exclusion principle in phase space. Then, $S[\rho]$ can be obtained by a standard combinatorial analysis respecting this exclusion principle. The equilibrium states of Eq. (35) are given by a Fermi-like distribution in physical space

$$
\rho=\frac{\sigma_{0}}{1+\lambda e^{-\frac{\chi}{D} c}}
$$

which maximizes the effective free energy (36) at fixed mass.

It is clear on qualitative grounds that the system of equations (35)-(33) will display exactly the same phenomena as the system of self-gravitating Brownian fermions (at least in dimension $D=3$ ) [31]. In particular, by tuning the mass of the system, or more generally the dimensionless parameter $\eta=\frac{\lambda \chi M}{4 \pi D R}$, we can describe an hysteretic cycle similar to the one depicted in Fig. 4. Above a critical mass, blow-up occurs until finite size effects come into play and arrest the collapse. Then, by slowly decreasing the mass of the aggregate, we reach a critical point $\eta_{*}$ at which an explosion sets in. This occurs when diffusion prevails over chemotactic drift. This hysteretic cycle has never been reported in the chemotactic literature and we can wonder whether it could be observed in biological experiments. In any case, the constraint $\rho \leq \sigma_{0}$ implied by Eq. (35) regularizes the problem and prevents unphysical infinities.

On the other hand, as pointed out by Keller \& Segel [8], the diffusion coefficient of amoebae can depend on the density $\rho$, leading to a situation of anomalous diffusion. For example, the case where the diffusion coefficient is a power law of the density has been investigated in [20]. Moreover, the relation between the concentration of amoebae and acrasin may be more complex that simply given by the Poisson equation (33). For example, taking $b \neq 0$ in the original model, we obtain a relation of the form

$$
\Delta c-k_{S}^{2} c=-\lambda \rho
$$


where $k_{S}^{2}=b / D^{\prime}$. The second term is similar to the Debye shielding in plasma physics. These remarks motivate us to consider a larger class of drift-diffusion equations of the form

$$
\frac{\partial \rho}{\partial t}=\nabla \cdot\left\{D\left[\nabla \rho+\frac{\beta}{C^{\prime \prime}(\rho)} \nabla \int u\left(\mathbf{r}-\mathbf{r}^{\prime}\right) \rho\left(\mathbf{r}^{\prime}, t\right) d^{D} \mathbf{r}^{\prime}\right]\right\} .
$$

This generalized class of Fokker-Planck equations has been introduced by Chavanis [15, $32,16,30]$. They include an arbitrary diffusion coefficient $D(\mathbf{r}, t)$, an arbitrary convex function $C(\rho)$ and an arbitrary binary potential of interaction $u\left(\mathbf{r}-\mathbf{r}^{\prime}\right)$. Equation (39) can therefore provide a generalized model of chemotactic aggregation taking into account anomalous diffusion, stickening effects and shielding effects of various forms. This equation increases a Lyapunov functional

$$
J[\rho]=-\int C(\rho) d^{D} \mathbf{r}-\frac{1}{2} \beta \int \rho(\mathbf{r}, t) u\left(\mathbf{r}-\mathbf{r}^{\prime}\right) \rho\left(\mathbf{r}^{\prime}, t\right) d^{D} \mathbf{r} d^{D} \mathbf{r}^{\prime},
$$

which plays the role of a generalized free energy in an effective thermodynamical formalism. Finally, the stationary solutions of this equation are determined by the integrodifferential equation

$$
C^{\prime}(\rho)=-\beta \int u\left(\mathbf{r}-\mathbf{r}^{\prime}\right) \rho\left(\mathbf{r}^{\prime}\right) d^{D} \mathbf{r}-\alpha .
$$

In the limit of short range interactions, the non-local drift-diffusion equation (39) reduces to a form of Cahn-Hilliard equation

$$
\frac{\partial \rho}{\partial t}=\nabla \cdot\left\{\chi \nabla\left(\Delta \rho-V^{\prime}(\rho)\right)\right\}
$$

where $V(\rho)=-(2 / b \beta) C(\rho)-(a / b) \rho^{2}, \chi=\beta \frac{b}{2} \frac{D}{C^{\prime \prime}(\rho)}$ with $a=\int u(|\mathbf{x}|) d^{D} \mathbf{x}$ and $b=$ $\frac{1}{D} \int u(|\mathbf{x}|) x^{2} d^{D} \mathbf{x}[30,16]$. The Cahn-Hilliard equation has been extensively studied in the theory of phase ordering kinetics. In the chemotactic model, the short-range interaction limit is reached when $k \gg 1$, i.e. in a regime of high degradation rate. The Cahn-Hilliard equation is known to develop "domain walls" and other morphological structures. It would be interesting to see whether such solutions can be constructed and observed in the context of bacterial aggregation.

The drift-diffusion equation (39) does not take into account memory effects. However, if we come back to the original Keller-Segel model, the concentration of acrasin $c$ is related to the concentration of amoeba $\rho$ by an equation of the form

$$
\frac{\partial c}{\partial t}=D^{\prime} \Delta c+a \rho-b c
$$

which involves a time derivative. Therefore, a more general model of chemotaxis is represented by the non-Markovian equation

$$
\frac{\partial \rho}{\partial t}=\nabla \cdot\left\{D\left[\nabla \rho+\frac{\beta}{C^{\prime \prime}(\rho)} \nabla \iint_{0}^{t} u\left(\mathbf{r}-\mathbf{r}^{\prime}, t-t^{\prime}\right) \rho\left(\mathbf{r}^{\prime}, t^{\prime}\right) d^{D} \mathbf{r}^{\prime} d t^{\prime}\right]\right\}
$$

taking into account delay effects [30].

7. Hyperbolic models. Recent experiments of in vitro formation of blood vessels show that cells randomly spread on a gel matrix autonomously organize to form a connected vascular network that is interpreted as the beginning of a vasculature. This phenomenon 
is responsible of angiogenesis, a major factor for the growth of tumors. These networks cannot be explained by the above parabolic models that lead to pointwise blow-up. However, they can be recovered by certain hyperbolic models. In particular, Gamba et al. [33] describe the evolution of the cell population by the equations

$$
\begin{gathered}
\frac{\partial \rho}{\partial t}+\nabla \cdot(\rho \mathbf{u})=0, \quad \frac{\partial \mathbf{u}}{\partial t}+\mathbf{u} \cdot \nabla \mathbf{u}=-\nabla h(\rho)+\mu \nabla c \\
\frac{\partial c}{\partial t}=D^{\prime} \Delta c+a \rho-b c
\end{gathered}
$$

and show, through extensive numerical simulations, that these equations develop network patterns that are in good agreement with experimental results. Additional numerical simulations are performed in [34] (see Fig. 7).

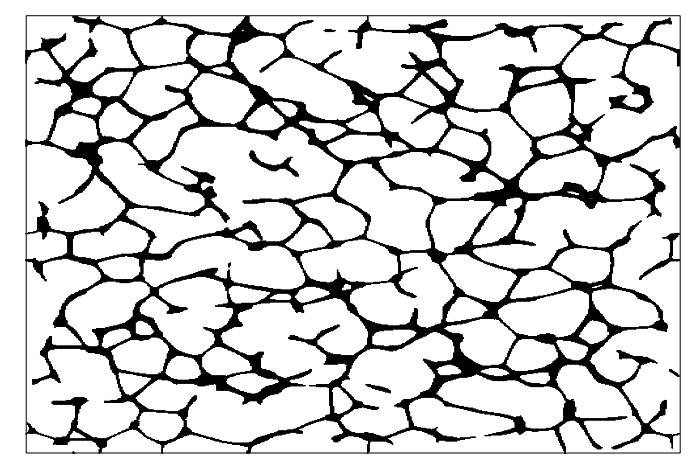

Fig. 7. Formation of a network in the hyperbolic model (45)-(46) of chemotactic aggregation [34]. We thank F. Filbet for having provided this figure.

It can be noted that these equations are similar to the Euler-Jeans equations

$$
\begin{gathered}
\frac{\partial \rho}{\partial t}+\nabla \cdot(\rho \mathbf{u})=0, \quad \frac{\partial \mathbf{u}}{\partial t}+\mathbf{u} \cdot \nabla \mathbf{u}=-\nabla h(\rho)-\nabla \Phi, \\
\Delta \Phi=4 \pi G \rho
\end{gathered}
$$

considered in the astrophysical literature (in that context, $h(\rho)$ is the enthalpy). These equations also describe the emergence of network-like patterns in the large-scale distribution of masses in the universe (in that case we need to account for the expansion of the universe). This is another aspect of the beautiful analogy between astrophysics and biology.

The connexion between parabolic and hyperbolic models can be made explicit by introducing a friction force $-\xi \mathbf{u}$ in the momentum equation. This friction term can take into account the influence of the gel matrix on the motion of cells in biology or the friction with a gas in the case of dust particles moving through the solar nebula [6]. In the high friction limit, or for sufficiently large times $t \gg \xi^{-1}$, the inertia of the particles can be neglected and the parabolic models (15) and (32) are recovered. 
8. The Hamiltonian $N$-vortex problem. We finally conclude this discussion by showing that two-dimensional vortices in fluid mechanics also share some analogies with selfgravitating systems and bacterial populations. Consider a system of $N$ point vortices in $2 \mathrm{D}$ hydrodynamics. The equations of motion can be cast in a Hamiltonian form

$$
\gamma \frac{d x_{i}}{d t}=\frac{\partial H}{\partial y_{i}}, \quad \gamma \frac{d y}{d t}=-\frac{\partial H}{\partial x_{i}},
$$

where $H$ is the Hamiltonian

$$
H=-\frac{1}{2 \pi} \sum_{i<j} \gamma^{2} \ln \left|\mathbf{r}_{i}-\mathbf{r}_{j}\right|
$$

and $\gamma$ the circulation of a point vortex. Since the total energy is conserved, the correct statistical description is the microcanonical ensemble. The statistical mechanics of point vortices was initiated by Onsager [35]. He showed the existence of negative temperature states $\beta<0$ at which point vortices cluster in macrovortices. This property is related to the emergence of large-scale vortices in atmospheric flows like, e.g., Jupiter's great red spot.

The statistical equilibrium state of a system of point vortices has been determined by Joyce \& Mongomery [36] in a meanfield approximation. It is obtained by maximizing the Boltzmann entropy

$$
S=-\int \omega \ln \omega d^{2} \mathbf{r}
$$

at fixed circulation $\Gamma=\int \omega d^{2} \mathbf{r}$ and energy $E=\frac{1}{2} \int \omega \psi d^{2} \mathbf{r}$, where $\omega$ is the vorticity and $\psi$ the streamfunction. This leads to the Boltzmann distribution

$$
\omega=A e^{-\beta \psi},
$$

where the stream-function $\psi$ is determined self-consistently by substituting the relation (52) in the Poisson equation

$$
\omega=-\Delta \psi
$$

and solving the resulting Boltzmann-Poisson system. We note that the equilibrium distribution of point vortices at negative temperatures $(52)(53)$ is similar to the equilibrium distribution of stars (8) (4) and bacteries (34) (33). Therefore, there is a deep analogy between two-dimensional vortices, stellar systems and biological clusters as first emphasized in $[37,15]$.

A kinetic theory of point vortices has been developed by Chavanis [38], using methods inspired from stellar dynamics and plasma physics. Starting from the $N$-body Liouville equation, using projection operator technics and considering axisymmetric flows, he obtained a kinetic equation of the form

$$
\frac{\partial \omega}{\partial t}=-\frac{\gamma}{4 r} \frac{\partial}{\partial r} \int_{0}^{+\infty} r_{1} d r_{1} \delta\left(\Omega-\Omega_{1}\right) \ln \left[1-\left(\frac{r_{<}}{r_{>}}\right)^{2}\right]\left\{\frac{1}{r} \omega_{1} \frac{\partial \omega}{\partial r}-\frac{1}{r_{1}} \omega \frac{\partial \omega_{1}}{\partial r_{1}}\right\}
$$

where $\Omega=\Omega(r, t), \Omega_{1}=\Omega\left(r_{1}, t\right)$ and $r_{>}$(resp. $r_{<}$) is the biggest (resp. smallest) of $r$ and $r_{1}$. This equation governs the evolution of the average vorticity $\omega(\mathbf{r}, t)=\gamma \rho(\mathbf{r}, t)$ 
which is proportional to the local density $\rho(\mathbf{r}, t)$ of point vortices. The angular velocity $\Omega(r, t)=\left\langle V_{\theta}\right\rangle(r, t) / r$ is related to the vorticity by

$$
\omega=\frac{1}{r} \frac{\partial}{\partial r}\left(\Omega r^{2}\right)
$$

More general kinetic equations, accounting for memory effects, have also been derived in [38] but they are difficult to study. Equation (54) is the counterpart of the Landau equation (5) in gravitational dynamics. The evolution of the vortex cloud is due to a condition of resonance encapsulated in the $\delta$-function. This resonance takes into account distant collisions between point vortices and is manifest only if the profile of angular velocity is non-monotonic (otherwise the collision term vanishes). The kinetic equation (54) conserves all the constraints of the $N$-vortex system (circulation, energy, impulse and angular momentum) and increases the Boltzmann entropy (51). However, as discussed in [38], this kinetic equation does not relax towards the thermal equilibrium state (52) predicted by the statistical mechanics of Joyce \& Montgomery [36]. The reason is not well-understood. This may be a signature of the lack of ergodicity in the point vortex gas. This may also correspond to the break-up of the assumptions leading to Eq. (54). Non-trivial three body correlations may be necessary to produce further evolution [38]. However, these correlations will be manifest on longer timescales and the system can remain frozen in a sort of metastable state for a very long time.

9. Brownian vortices. As in the case of self-gravitating systems, it is of conceptual interest to compare the microcanonical evolution of a Hamiltonian system of point vortices to a canonical model. We thus introduce formally a system of $N$ "Brownian vortices" (the counterpart of self-gravitating Brownian particles) interacting through a logarithmic potential in two dimensions. By definition, this system is described by the Langevin equations

$$
\frac{d \mathbf{r}_{i}}{d t}=-\mathbf{z} \times \nabla_{i} U\left(\mathbf{r}_{1}, \ldots, \mathbf{r}_{N}\right)-\xi \nabla_{i} U\left(\mathbf{r}_{1}, \ldots, \mathbf{r}_{N}\right)+\sqrt{2 D} \mathbf{R}_{i}(t),
$$

where $-\xi \nabla_{i} U\left(\mathbf{r}_{1}, \ldots, \mathbf{r}_{N}\right)$ is a drift and $\mathbf{R}_{i}(t)$ is a white noise. The potential of interaction is given by $U\left(\mathbf{r}_{1}, \ldots, \mathbf{r}_{N}\right)=\sum_{i<j} u\left(\mathbf{r}_{i}-\mathbf{r}_{j}\right)$ where $u\left(\mathbf{r}_{i}-\mathbf{r}_{j}\right)=-\frac{1}{2 \pi} \ln \left|\mathbf{r}_{i}-\mathbf{r}_{j}\right|$. Finally, we define the inverse temperature $\beta=1 / T$ through the Einstein relation $\xi=D \beta$. Since the temperature $T$ is fixed, the Brownian vortex model has a rigorous canonical structure. This model can be seen as a generalization of the stochastic model of Chavanis [39, 38] which describes the evolution of a single test vortex in a bath of field vortices at statistical equilibrium (this is the counterpart of the Chandrasekhar [14] stochastic model in stellar dynamics). In that case, the drift and the diffusion arise from discrete interactions with the field vortices which play the role of a thermal bath. Equation (56) without the first term can also provide a stochastic model of chemotaxis.

Starting from the $N$-body Fokker-Planck equation, implementing a mean-field approximation and following the lines of Appendix B, we find that the average vorticity $\omega(\mathbf{r}, t)=\gamma \rho(\mathbf{r}, t)$ satisfies a Fokker-Planck equation of the form

$$
\frac{\partial \omega}{\partial t}+\mathbf{u} \cdot \nabla \omega=\nabla \cdot[D(\nabla \omega+\beta \gamma \omega \nabla \psi)]
$$


coupled to the Poisson equation

$$
\Delta \psi=-\omega
$$

The drift-diffusion equation (57) conserves circulation and increases the free energy $J=S-\beta E$ (canonical description). Moreover, the Boltzmann distribution (52) is the only stationary solution of this equation contrary to the kinetic equation (54) valid for the Hamiltonian system. We note the analogy with the Smoluchowski-Poisson system (15)-(16) and (32)-(33) describing self-gravitating Brownian particles and bacterial populations. In the case of Brownian point vortices, the Fokker-Planck equation directly takes the form of a drift-diffusion equation while for self-gravitating Brownian particles, the Smoluchowski equation is obtained in a high friction limit where the motion of the particles is overdamped. We also emphasize the difference of behaviours between the Brownian vortex model (56)-(57) and the Hamiltonian vortex model (49)-(54). In the canonical ensemble in $D=2$, there exists a critical inverse temperature $\beta_{c}=-\frac{8 \pi}{\gamma \Gamma}$ below which a system of Brownian vortices collapses [19]. By contrast, in the microcanononical ensemble, there is no collapse in $D=2$. This is related to the fact that, in two dimensions, there exists a maximum entropy state for all values of energies while the free energy has a minimum only for $\beta>\beta_{c}$ (the states with $\beta<\beta_{c}$ are not accessible in the microcanonical ensemble) [37]. Therefore, a naive canonical description of point vortices in two dimensions does not describe the true Hamiltonian system correctly. This is a manifestation of ensemble inequivalence for systems with long-range interactions, such as point vortices.

10. Violent relaxation and metaequilibrium states. In the thermodynamical limit $N \rightarrow+\infty$ with $\eta=\beta N \gamma^{2}$ fixed, appropriate to point vortices, the collision term appearing in the kinetic equation (54) is of order $N^{-1}$. It represents therefore the first order correction to the Vlasov limit [37]. However, in general, the "collisions" are negligible in the point vortex gas and the evolution of the vorticity/density is described for timescales of interest by the 2D Euler-Poisson system

$$
\begin{gathered}
\frac{\partial \omega}{\partial t}+\mathbf{u} \cdot \nabla \omega=0, \\
\Delta \psi=-\omega .
\end{gathered}
$$

This corresponds to the Vlasov regime in stellar dynamics and plasma physics. These equations can be derived from the Liouville equation by assuming that the $N$-body distribution function factorizes as $N$ one-body distribution functions, which is justified in the limit $N \rightarrow+\infty$ (for fixed $t$ ) [38]. The Euler equation also describes the inviscid evolution of continuous vorticity flows in two dimensions (see, e.g., [37]).

It is known that the 2D Euler-Poisson system develops a complicated mixing process leading to a metaequilibrium state on the coarse-grained scale. This process is called violent relaxation. A small-scale parametrization of the 2D Euler equation can be attempted by using thermodynamical arguments [40] and kinetic theory [41]. Similar arguments can be developed for the Vlasov-Poisson system [18]. In the two-levels approximation of their statistical theory, Robert \& Sommeria [40] have proposed a parametrization of the form

$$
\frac{\partial \bar{\omega}}{\partial t}+\mathbf{u} \cdot \nabla \bar{\omega}=\nabla \cdot\left[D\left(\nabla \bar{\omega}+\beta(t)\left(\bar{\omega}-\sigma_{-1}\right)\left(\sigma_{1}-\bar{\omega}\right) \nabla \psi\right)\right]
$$


where $\bar{\omega}$ and $\psi$ are coupled through the Poisson equation (60) and $\beta(t)$ evolves with time so as to satisfy the conservation of energy $\dot{E}=0$. This equation respects the constraints $\sigma_{-1} \leq \bar{\omega} \leq \sigma_{1}$ imposed by the Euler equation on the coarse-grained vorticity and converges towards the equilibrium distribution

$$
\bar{\omega}=\sigma_{-1}+\frac{\sigma_{1}-\sigma_{-1}}{1+\lambda e^{\beta\left(\sigma_{1}-\sigma_{-1}\right) \psi}},
$$

which maximizes the mixing entropy

$$
S=-\int[p \ln p+(1-p) \ln (1-p)] d^{2} \mathbf{r},
$$

with $\bar{\omega}=p \sigma_{1}+(1-p) \sigma_{-1}$, at fixed circulation and energy. An example of relaxation towards statistical equilibrium is shown in Fig. 8 in relation with the formation of largescale vortices in the jovian atmosphere [42]. We note the analogy between Eq. (61) and the model (35) proposed for the chemotactic aggregration of bacterial populations (consider in particular the case $\sigma_{-1}=0$ ). One important difference is that $\beta$ in Eq. (61) is not fixed but evolves with time so as to satisfy the conservation of energy. Hence, Eq. (61) describes a microcanonical situation while Eq. (35) can be associated with a canonical situation $[15,30]$. The well-posedness of Eq. (61) has been established in $[43,44]$.
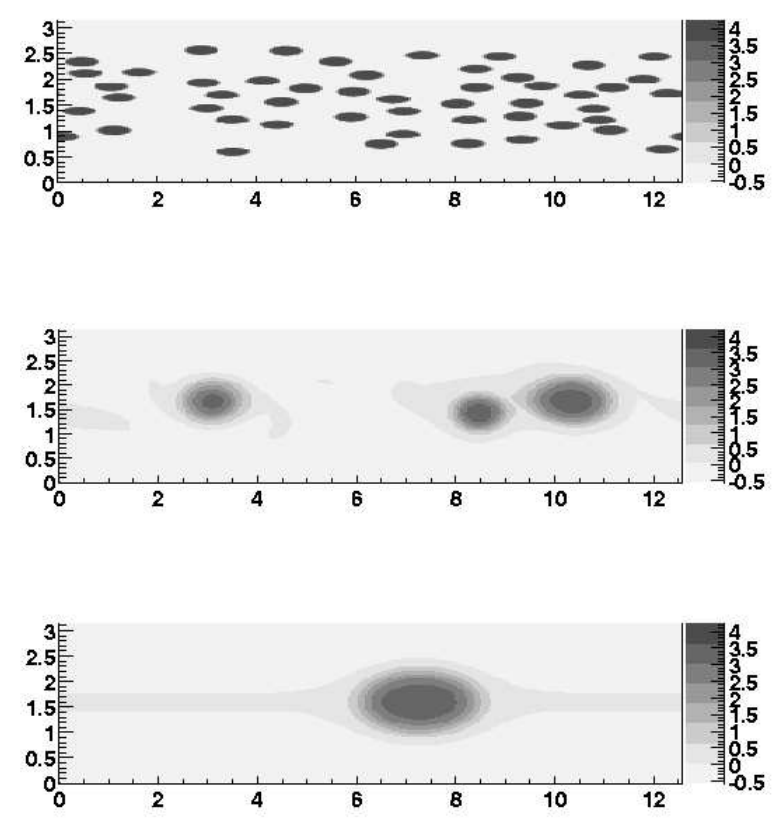

Fig. 8. Relaxation towards statistical equilibrium in a QG model of Jupiter's great red spot and other jovian vortices (white ovals), using the parameterization (61) [42]. We thank F. Bouchet for having provided this figure.

In the quasi-geostrophic approximation appropriate to geophysical fluid dynamics [45], Eqs. (59) and (61) remain valid provided that the vorticity $\omega$ is replaced by the potential 
vorticity $q$ which is related to $\psi$ by a relation of the form

$$
q=-\Delta \psi+\frac{1}{R^{2}} \psi+h(y)
$$

In this equation, $h(y)$ represents a topography and $R$ is the so-called Rossby radius. It takes into account the deformation of the surface of the fluid. This equation should be compared with the similar equation (38) in the chemotactic problem. In the limit of small Rossby radius $R \rightarrow 0$, the relaxation equation (61) becomes equivalent to the Cahn-Hilliard equation as discussed in [46]. In that case, its stationary solutions describe "domain walls". These solutions precisely account for the jet structure of Jupiter's great red spot [47].

The parametrization of Robert \& Sommeria [40] is complicated to implement in the case of realistic initial conditions with a large number of vorticity levels. In order to go beyond the two-levels approximation while leaving the problem tractable, Chavanis $[15,46]$ has proposed an equation of the form

$$
\frac{\partial q}{\partial t}+\mathbf{u} \cdot \nabla q=\nabla \cdot\left\{D\left[\nabla q+\frac{\beta(t)}{C^{\prime \prime}(q)} \nabla \psi\right]\right\},
$$

where $C$ is a convex function. The relation between the potential vorticity and the streamfunction is written in the general form

$$
\psi(\mathbf{r}, t)=\int u\left(\mathbf{r}-\mathbf{r}^{\prime}\right) q\left(\mathbf{r}^{\prime}, t\right) d^{2} \mathbf{r}^{\prime}
$$

The relaxation equation (65) provides a simplified parametrization of the 2D EulerPoisson system, where the function $C(q)$ has to be adapted to the physical context (ocean dynamics, jovian atmosphere,...). Alternatively, it can be used as a numerical algorithm to construct arbitrary nonlinearly dynamically stable stationary solutions of the $2 \mathrm{D}$ Euler equation, specified by a relationship $C^{\prime}(q)=-\beta \psi-\alpha$, in an attempt to reproduce observed phenomena $[15,46]$.

11. Conclusion. In this paper, we have emphasized the analogy between self gravitating systems, two-dimensional vortices and bacterial populations. This completes the discussion initiated in $[37,15]$. We have introduced or quoted different types of models and kinetic equations. Some models have a rigorous physical justification. Others have a more academic or conceptual interest. The common point between these models is the long-range nature of the interactions leading to non-local kinetic equations or non-local Fokker-Planck equations $[15,32,30]$. They correspond either to a microcanonical situation (fixed energy) or a canonical one (fixed temperature). These equations have a very rich mathematical and physical structure associated with phase transitions, blow-up phenomena and morphogenesis. It is clear that these equations have a "fundamental" character and that they deserve to be studied in detail. This systematic study has been undertaken by the authors in $[4,19,20,21,22,27]$.

Acknowledgements. One of us (P.-H. C) would like to thank P. Laurençot for interesting discussions concerning hyperbolic models of chemotaxis. 
Appendix A: The canonical statistical equilibrium state. In this Appendix, we use the equilibrium BBGKY hierarchy and a mean-field approximation to determine the statistical equilibrium state of particles in interaction in the canonical ensemble (a similar approach can be developped in the microcanonical ensemble [48]). The canonical distribution in physical space is given by

$$
P_{N}\left(\mathbf{r}_{1}, \ldots, \mathbf{r}_{N}\right)=\frac{1}{Z(\beta)} e^{-\beta U\left(\mathbf{r}_{1}, \ldots, \mathbf{r}_{N}\right)},
$$

where $Z(\beta)$ is the partition function

$$
Z(\beta)=\int e^{-\beta U\left(\mathbf{r}_{1}, \ldots, \mathbf{r}_{N}\right)} d^{D} \mathbf{r}_{1} \ldots d^{D} \mathbf{r}_{N}
$$

In practice, we are not interested by the $N$-body distribution function $P_{N}\left(\mathbf{r}_{1}, \ldots, \mathbf{r}_{N}\right)$ but rather by the reduced densities such as

$$
\begin{gathered}
P_{1}(\mathbf{r})=\int P_{N}\left(\mathbf{r}, \mathbf{r}_{2}, \ldots, \mathbf{r}_{N}\right) d^{D} \mathbf{r}_{2} \ldots d^{D} \mathbf{r}_{N} \\
P_{2}\left(\mathbf{r}, \mathbf{r}^{\prime}\right)=\int P_{N}\left(\mathbf{r}, \mathbf{r}^{\prime}, \mathbf{r}_{3}, \ldots, \mathbf{r}_{N}\right) d^{D} \mathbf{r}_{3} \ldots d^{D} \mathbf{r}_{N} .
\end{gathered}
$$

Assuming that the particles are identical, the average density in $\mathbf{r}$ is

$$
\rho(\mathbf{r})=\left\langle\sum_{i} \delta\left(\mathbf{r}_{i}-\mathbf{r}\right)\right\rangle=N P_{1}(\mathbf{r}) .
$$

Taking the derivative of Eq. (67) with respect to $\mathbf{r}_{1}$, we get

$$
\frac{\partial P_{N}}{\partial \mathbf{r}_{1}}\left(\mathbf{r}_{1}, \ldots, \mathbf{r}_{N}\right)=-\beta P_{N} \frac{\partial U}{\partial \mathbf{r}_{1}}\left(\mathbf{r}_{1}, \ldots, \mathbf{r}_{N}\right) .
$$

We now integrate over $\mathbf{r}_{2}, \ldots, \mathbf{r}_{N}$ to obtain

$$
\frac{\partial P_{1}}{\partial \mathbf{r}_{1}}\left(\mathbf{r}_{1}\right)=-\beta \int \prod_{i=2}^{N} d^{D} \mathbf{r}_{i} P_{N}\left(\mathbf{r}_{1}, \ldots, \mathbf{r}_{N}\right) \frac{\partial U}{\partial \mathbf{r}_{1}}\left(\mathbf{r}_{1}, \ldots, \mathbf{r}_{N}\right) .
$$

For a binary potential of interaction

$$
U\left(\mathbf{r}_{1}, \ldots, \mathbf{r}_{N}\right)=\sum_{i<j} u\left(\mathbf{r}_{i}-\mathbf{r}_{j}\right), \quad u(\mathbf{r})=u(-\mathbf{r}),
$$

the foregoing expression can be rewritten

$$
\frac{\partial P_{1}}{\partial \mathbf{r}_{1}}\left(\mathbf{r}_{1}\right)=-\beta \sum_{j=2}^{N} \int \prod_{i=2}^{N} d^{D} \mathbf{r}_{i} P_{N}\left(\mathbf{r}_{1}, \ldots, \mathbf{r}_{N}\right) \frac{\partial u}{\partial \mathbf{r}_{1}}\left(\mathbf{r}_{1}-\mathbf{r}_{j}\right) .
$$

Since the particles are identical

$$
\frac{\partial P_{1}}{\partial \mathbf{r}_{1}}\left(\mathbf{r}_{1}\right)=-\beta(N-1) \int \prod_{i=2}^{N} d^{D} \mathbf{r}_{i} P_{N}\left(\mathbf{r}_{1}, \ldots, \mathbf{r}_{N}\right) \frac{\partial u}{\partial \mathbf{r}_{1}}\left(\mathbf{r}_{1}-\mathbf{r}_{2}\right)
$$

or, equivalently,

$$
\frac{\partial P_{1}}{\partial \mathbf{r}_{1}}\left(\mathbf{r}_{1}\right)=-\beta(N-1) \int d^{D} \mathbf{r}_{2} P_{2}\left(\mathbf{r}_{1}, \mathbf{r}_{2}\right) \frac{\partial u}{\partial \mathbf{r}_{1}}\left(\mathbf{r}_{1}-\mathbf{r}_{2}\right)
$$


For $N \gg 1$ such that $\beta N u \sim 1$, which defines the proper thermodynamic limit of this nonextensive system, it can be shown [48] that the mean field approximation is exact, i.e.

$$
P_{2}\left(\mathbf{r}_{1}, \mathbf{r}_{2}\right)=P_{1}\left(\mathbf{r}_{1}\right) P_{1}\left(\mathbf{r}_{2}\right)+O(1 / N) .
$$

Thus, Eq. (77) becomes

$$
\frac{\partial P_{1}}{\partial \mathbf{r}_{1}}\left(\mathbf{r}_{1}\right)=-\beta N P_{1}\left(\mathbf{r}_{1}\right) \frac{\partial}{\partial \mathbf{r}_{1}} \int d^{D} \mathbf{r}_{2} P_{1}\left(\mathbf{r}_{2}\right) u\left(\mathbf{r}_{1}-\mathbf{r}_{2}\right) .
$$

Introducing the mean-field potential

$$
\Phi(\mathbf{r})=\int d^{D} \mathbf{r}^{\prime} \rho\left(\mathbf{r}^{\prime}\right) u\left(\mathbf{r}-\mathbf{r}^{\prime}\right)
$$

produced by the smooth density $\rho(\mathbf{r})=N P_{1}(\mathbf{r})$, we obtain

$$
\frac{\partial P_{1}}{\partial \mathbf{r}}(\mathbf{r})=-\beta P_{1}(\mathbf{r}) \frac{\partial \Phi}{\partial \mathbf{r}}(\mathbf{r})
$$

After integration, we find that the density of particles is given by the Boltzmann distribution

$$
\rho(\mathbf{r})=A e^{-\beta \Phi(\mathbf{r})},
$$

where $\Phi$ is related to $\rho$ via Eq. (80). Hence, Eq. (82) must be regarded as an integrodifferential equation.

We now consider the canonical distribution in phase space

$$
P_{N}\left(\mathbf{r}_{1}, \mathbf{v}_{1}, \ldots, \mathbf{r}_{N}, \mathbf{v}_{N}\right)=\frac{1}{Z(\beta)} e^{-\beta\left\{\sum_{i=1}^{N} \frac{v_{i}^{2}}{2}+U\left(\mathbf{r}_{1}, \ldots, \mathbf{r}_{N}\right)\right\}} .
$$

Noting that the velocity distribution is Gaussian, we find that Eq. (82) is now replaced by the Maxwell-Boltzmann distribution

$$
f(\mathbf{r}, \mathbf{v})=A e^{-\beta\left(\frac{v^{2}}{2}+\Phi(\mathbf{r})\right)},
$$

where, again, $\Phi$ is related to $\rho$ via Eq. (80). The distribution function (84) can also be obtained by minimizing the Boltzmann free energy $F_{B}[f]$ at fixed mass $M$ and temperature $T$ (see Sec. 3). This method provides a condition of thermodynamical stability $\delta^{2} F \geq 0$, which is not captured by the equilibrium BBGKY hierarchy. Indeed, the thermodynamical stability is related to the dynamical stability with respect to the $N$-body Fokker-Planck equation (see Appendix B) corresponding to the time dependent BBGKY hierarchy (see also [15]).

\section{Appendix B: Brownian particles in interaction}

B1. Stochastic processes in physical space. In this Appendix, we derive the mean-field Fokker-Planck equation (15) describing a gas of Brownian particles in interaction, using the method of Martzel \& Aslangul [49, 50]. First, we consider a system of Brownian particles in physical space. The state of the system is completely specified by the $N$-body distribution function $P_{N}\left(\mathbf{r}_{1}, \ldots, \mathbf{r}_{N}, t\right)$ which gives the density probability of finding at time $t$ the first particle in $\mathbf{r}_{1}$, the second in $\mathbf{r}_{2}$ etc. We assume that the evolution of the system is governed by the stochastic process

$$
\frac{d \mathbf{r}_{i}}{d t}=-\mu \nabla_{i} U\left(\mathbf{r}_{1}, \ldots, \mathbf{r}_{N}\right)+\sqrt{2 D_{*}} \mathbf{R}_{i}(t)
$$


where $\mathbf{R}_{i}(t)$ is a white noise satisfying $\left\langle\mathbf{R}_{i}(t)\right\rangle=\mathbf{0}$ and $\left\langle R_{a, i}(t) R_{b, j}\left(t^{\prime}\right)\right\rangle=\delta_{i j} \delta_{a b} \delta\left(t-t^{\prime}\right)$, where $a, b=1, \ldots, D$ refer to the coordinates of space and $i, j=1, \ldots, N$ to the particles. The particles interact via the potential $U\left(\mathbf{r}_{1}, \ldots, \mathbf{r}_{N}\right)=\sum_{i<j} u\left(\mathbf{r}_{i}-\mathbf{r}_{j}\right)$.

For a general Markovian process, we have

$$
\begin{array}{r}
P_{N}\left(\mathbf{r}_{1}, \ldots, \mathbf{r}_{N}, t+\Delta t\right)=\int d^{D}\left(\Delta \mathbf{r}_{1}\right) \\
\ldots d^{D}\left(\Delta \mathbf{r}_{N}\right) P_{N}\left(\mathbf{r}_{1}-\Delta \mathbf{r}_{1}, \ldots, \mathbf{r}_{N}-\Delta \mathbf{r}_{N}, t\right) \\
\times w\left(\mathbf{r}_{1}-\Delta \mathbf{r}_{1}, \ldots, \mathbf{r}_{N}-\Delta \mathbf{r}_{N} \mid \Delta \mathbf{r}_{1}, \ldots, \Delta \mathbf{r}_{N}\right)
\end{array}
$$

where $w$ is the transition probability. Expanding the right hand side in Taylor series and introducing the Kramers-Moyal moments

$$
\begin{aligned}
M_{n_{1} \ldots n_{N}}\left(\mathbf{r}_{1}, \ldots,\right. & \left.\mathbf{r}_{N}\right)=\lim _{\Delta t \rightarrow 0} \frac{1}{\Delta t n_{1} ! \ldots n_{N} !} \int d^{D}\left(\Delta \mathbf{r}_{1}\right) \ldots d^{D}\left(\Delta \mathbf{r}_{N}\right) \\
& \times\left(-\Delta \mathbf{r}_{1}\right)^{n_{1}} \ldots\left(-\Delta \mathbf{r}_{N}\right)^{n_{N}} w\left(\mathbf{r}_{1}, \ldots, \mathbf{r}_{N} \mid \Delta \mathbf{r}_{1}, \ldots, \Delta \mathbf{r}_{N}\right),
\end{aligned}
$$

we get the $N$-body Fokker-Planck equation

$$
\frac{\partial P_{N}}{\partial t}=\sum_{n_{1} \ldots n_{N}} \frac{\partial^{n_{1}}}{\partial \mathbf{r}_{1}^{n_{1}}} \ldots \frac{\partial^{n_{N}}}{\partial \mathbf{r}_{N}^{n_{N}}}\left\{M_{n_{1} \ldots n_{N}} P_{N}\right\}
$$

where the sum runs over all indices such that $\sum_{i} n_{i} \geq 1$. For the stochastic process (85), only a few moments do not vanish, namely

$$
\begin{gathered}
M_{0 \ldots n_{i}=1 \ldots 0}=\mu \nabla_{i} U\left(\mathbf{r}_{1}, \ldots, \mathbf{r}_{N}\right), \\
M_{0 \ldots n_{i}=2 \ldots 0}=D_{*} .
\end{gathered}
$$

Substituting these results in the $N$-body Fokker-Planck equation (88), we obtain

$$
\frac{\partial P_{N}}{\partial t}=\sum_{i=1}^{N} \frac{\partial}{\partial \mathbf{r}_{i}}\left[D_{*} \frac{\partial P_{N}}{\partial \mathbf{r}_{i}}+\mu P_{N} \frac{\partial}{\partial \mathbf{r}_{i}} U\left(\mathbf{r}_{1}, \ldots, \mathbf{r}_{N}\right)\right] .
$$

We note that the stationary solutions of this equation correspond to the canonical equilibrium distribution (67) provided that $\mu=D_{*} \beta$ (Einstein relation). Therefore, a gas of Brownian particles in interaction has a rigorous canonical structure. Integrating over $\mathbf{r}_{2}, \ldots, \mathbf{r}_{N}$, we obtain

$$
\frac{\partial P_{1}}{\partial t}=\frac{\partial}{\partial \mathbf{r}_{1}}\left[D_{*} \frac{\partial P_{1}}{\partial \mathbf{r}_{1}}+\mu(N-1) \int d^{D} \mathbf{r}_{2} P_{2}\left(\mathbf{r}_{1}, \mathbf{r}_{2}, t\right) \frac{\partial}{\partial \mathbf{r}_{1}} u\left(\mathbf{r}_{1}-\mathbf{r}_{2}\right)\right] .
$$

Now, implementing a mean-field approximation which is valid in the proper thermodynamic limit $N \gg 1$ with $\beta N u \sim 1[48]$ :

$$
P_{2}\left(\mathbf{r}_{1}, \mathbf{r}_{2}, t\right)=P_{1}\left(\mathbf{r}_{1}, t\right) P_{1}\left(\mathbf{r}_{2}, t\right)+O(1 / N),
$$

the foregoing equation can be rewritten

$$
\frac{\partial P_{1}}{\partial t}=\frac{\partial}{\partial \mathbf{r}_{1}}\left[D_{*} \frac{\partial P_{1}}{\partial \mathbf{r}_{1}}+\mu P_{1} \frac{\partial \Phi}{\partial \mathbf{r}_{1}}\right]
$$

which is clearly the same as

$$
\frac{\partial \rho}{\partial t}=\nabla\left[D_{*}(\nabla \rho+\beta \rho \nabla \Phi)\right],
$$

where $\Phi(\mathbf{r}, t)$ is related to $\rho(\mathbf{r}, t)$ as in Eq. (80). 
B2. Stochastic processes in phase space. We now consider the generalization of the preceding problem to the case of $N$-coupled Brownian equations in phase space described by the stochastic process

$$
\frac{d \mathbf{r}_{i}}{d t}=\mathbf{v}_{i}, \quad \frac{d \mathbf{v}_{i}}{d t}=-\xi \mathbf{v}_{i}-\nabla_{i} U\left(\mathbf{r}_{1}, \ldots, \mathbf{r}_{N}\right)+\sqrt{2 D} \mathbf{R}_{i}(t)
$$

where $\mathbf{R}_{i}(t)$ is a white noise. We start from the Markov process,

$$
\begin{array}{r}
P_{N}\left(\mathbf{r}_{1}, \mathbf{v}_{1}, \ldots, \mathbf{r}_{N}, \mathbf{v}_{N}, t+\Delta t\right)=\int d^{D}\left(\Delta \mathbf{r}_{1}\right) d^{D}\left(\Delta \mathbf{v}_{1}\right) \ldots d^{D}\left(\Delta \mathbf{r}_{N}\right) d^{D}\left(\Delta \mathbf{v}_{N}\right) \\
\times P_{N}\left(\mathbf{r}_{1}-\Delta \mathbf{r}_{1}, \mathbf{v}_{1}-\Delta \mathbf{v}_{1}, \ldots, \mathbf{r}_{N}-\Delta \mathbf{r}_{N}, \mathbf{v}_{N}-\Delta \mathbf{v}_{N}, t\right) \\
\times w\left(\mathbf{r}_{1}-\Delta \mathbf{r}_{1}, \mathbf{v}_{1}-\Delta \mathbf{v}_{1}, \ldots, \mathbf{r}_{N}-\Delta \mathbf{r}_{N}, \mathbf{v}_{N}-\Delta \mathbf{v}_{N} \mid \Delta \mathbf{r}_{1}, \Delta \mathbf{v}_{1}, \ldots, \Delta \mathbf{r}_{N}, \Delta \mathbf{v}_{N}\right) .
\end{array}
$$

Now, the transition probability can be written

$$
\begin{array}{r}
w\left(\mathbf{r}_{1}, \mathbf{v}_{1}, \ldots, \mathbf{r}_{N}, \mathbf{v}_{N} \mid \Delta \mathbf{r}_{1}, \Delta \mathbf{v}_{1}, \ldots, \Delta \mathbf{r}_{N}, \Delta \mathbf{v}_{N}\right)= \\
\delta\left(\Delta \mathbf{r}_{1}-\mathbf{v}_{1} \Delta t\right) \ldots \delta\left(\Delta \mathbf{r}_{N}-\mathbf{v}_{N} \Delta t\right) \psi\left(\mathbf{r}_{1}, \mathbf{v}_{1}, \ldots, \mathbf{r}_{N}, \mathbf{v}_{N} \mid \Delta \mathbf{v}_{1}, \ldots, \Delta \mathbf{v}_{N}\right) .
\end{array}
$$

The integration over $\Delta \mathbf{r}_{1}, \ldots, \Delta \mathbf{r}_{N}$ is straightforward and yields

$$
\begin{array}{r}
P_{N}\left(\mathbf{r}_{1}, \mathbf{v}_{1}, \ldots, \mathbf{r}_{N}, \mathbf{v}_{N}, t+\Delta t\right)=\int d^{D}\left(\Delta \mathbf{v}_{1}\right) \ldots d^{D}\left(\Delta \mathbf{v}_{N}\right) \\
\times P_{N}\left(\mathbf{r}_{1}-\mathbf{v}_{1} \Delta t, \mathbf{v}_{1}-\Delta \mathbf{v}_{1}, \ldots, \mathbf{r}_{N}-\mathbf{v}_{N} \Delta t, \mathbf{v}_{N}-\Delta \mathbf{v}_{N}, t\right) \\
\times \psi\left(\mathbf{r}_{1}-\mathbf{v}_{1} \Delta t, \mathbf{v}_{1}-\Delta \mathbf{v}_{1}, \ldots, \mathbf{r}_{N}-\mathbf{v}_{N} \Delta t, \mathbf{v}_{N}-\Delta \mathbf{v}_{N} \mid \Delta \mathbf{v}_{1}, \ldots, \Delta \mathbf{v}_{N}\right),
\end{array}
$$

which is equivalent to

$$
\begin{array}{r}
P_{N}\left(\mathbf{r}_{1}+\mathbf{v}_{1} \Delta t, \mathbf{v}_{1}, \ldots, \mathbf{r}_{N}+\mathbf{v}_{N} \Delta t, \mathbf{v}_{N}, t+\Delta t\right)=\int d^{D}\left(\Delta \mathbf{v}_{1}\right) \ldots d^{D}\left(\Delta \mathbf{v}_{N}\right) \\
\times P_{N}\left(\mathbf{r}_{1}, \mathbf{v}_{1}-\Delta \mathbf{v}_{1}, \ldots, \mathbf{r}_{N}, \mathbf{v}_{N}-\Delta \mathbf{v}_{N}, t\right) \\
\times \psi\left(\mathbf{r}_{1}, \mathbf{v}_{1}-\Delta \mathbf{v}_{1}, \ldots, \mathbf{r}_{N}, \mathbf{v}_{N}-\Delta \mathbf{v}_{N} \mid \Delta \mathbf{v}_{1}, \ldots, \Delta \mathbf{v}_{N}\right) .
\end{array}
$$

Expanding the right hand side in Taylor series and introducing the Kramers-Moyal moments

$$
\begin{array}{r}
M_{n_{1} \ldots n_{N}}\left(\mathbf{r}_{1}, \mathbf{v}_{1}, \ldots, \mathbf{r}_{N}, \mathbf{v}_{N}\right)=\lim _{\Delta t \rightarrow 0} \frac{1}{\Delta t n_{1} ! \ldots n_{N} !} \int d^{D}\left(\Delta \mathbf{v}_{1}\right) \ldots d^{D}\left(\Delta \mathbf{v}_{N}\right) \\
\times\left(-\Delta \mathbf{v}_{1}\right)^{n_{1}} \ldots\left(-\Delta \mathbf{v}_{N}\right)^{n_{N}} \psi\left(\mathbf{r}_{1}, \mathbf{v}_{1}, \ldots, \mathbf{r}_{N}, \mathbf{v}_{N} \mid \Delta \mathbf{v}_{1}, \ldots, \Delta \mathbf{v}_{N}\right)
\end{array}
$$

we obtain the $N$-body Fokker-Planck equation

$$
\frac{\partial P_{N}}{\partial t}+\sum_{i=1}^{N} \mathbf{v}_{i} \frac{\partial P_{N}}{\partial \mathbf{r}_{i}}=\sum_{n_{1} \ldots n_{N}} \frac{\partial^{n_{1}}}{\partial \mathbf{v}_{1}^{n_{1}}} \ldots \frac{\partial^{n_{N}}}{\partial \mathbf{v}_{N}^{n_{N}}}\left\{M_{n_{1} \ldots n_{N}} P_{N}\right\},
$$

where the sum runs over all indices such that $\sum_{i} n_{i} \geq 1$. For the stochastic process (96), only a few moments do not vanish, namely

$$
\begin{gathered}
M_{0 \ldots n_{i}=1 \ldots 0}=\xi \mathbf{v}_{i}+\nabla_{i} U\left(\mathbf{r}_{1}, \ldots, \mathbf{r}_{N}\right) \\
M_{0 \ldots n_{i}=2 \ldots 0}=D
\end{gathered}
$$


Substituting these results in the $N$-body Fokker-Planck equation (102), we get

$$
\frac{\partial P_{N}}{\partial t}+\sum_{i=1}^{N}\left(\mathbf{v}_{i} \frac{\partial P_{N}}{\partial \mathbf{r}_{i}}+\mathbf{F}_{i} \frac{\partial P_{N}}{\partial \mathbf{v}_{i}}\right)=\sum_{i=1}^{N} \frac{\partial}{\partial \mathbf{v}_{i}}\left[D \frac{\partial P_{N}}{\partial \mathbf{v}_{i}}+\xi P_{N} \mathbf{v}_{i}\right]
$$

where $\mathbf{F}_{i}=-\nabla_{i} U\left(\mathbf{r}_{1}, \ldots, \mathbf{r}_{N}\right)$. We note that the stationary solutions of this equation correspond to the canonical equilibrium distribution (83) provided that $\xi=D \beta$ (Einstein relation). Integrating over $\mathbf{r}_{2}, \mathbf{v}_{2}, \ldots, \mathbf{r}_{N}, \mathbf{v}_{N}$, we obtain

$$
\frac{\partial P_{1}}{\partial t}+\mathbf{v}_{1} \frac{\partial P_{1}}{\partial \mathbf{r}_{1}}+\int \prod_{i=2}^{N} d^{D} \mathbf{r}_{i} d^{D} \mathbf{v}_{i} \mathbf{F}_{1} \frac{\partial P_{N}}{\partial \mathbf{v}_{1}}=\frac{\partial}{\partial \mathbf{v}_{1}}\left[D \frac{\partial P_{1}}{\partial \mathbf{v}_{1}}+\xi P_{1} \mathbf{v}_{1}\right]
$$

Now,

$$
\begin{array}{r}
\int \prod_{i=2}^{N} d^{D} \mathbf{r}_{i} d^{D} \mathbf{v}_{i} \mathbf{F}_{1} \frac{\partial P_{N}}{\partial \mathbf{v}_{1}}=-\int \sum_{j=2}^{N} \prod_{i=2}^{N} d^{D} \mathbf{r}_{i} d^{D} \mathbf{v}_{i} \frac{\partial u}{\partial \mathbf{r}_{1}}\left(\mathbf{r}_{1}-\mathbf{r}_{j}\right) \frac{\partial P_{N}}{\partial \mathbf{v}_{1}} \\
=-(N-1) \int d^{D} \mathbf{r}_{2} d^{D} \mathbf{v}_{2} \frac{\partial u}{\partial \mathbf{r}_{1}}\left(\mathbf{r}_{1}-\mathbf{r}_{2}\right) \frac{\partial P_{2}}{\partial \mathbf{v}_{1}}\left(\mathbf{r}_{1}, \mathbf{v}_{1}, \mathbf{r}_{2}, \mathbf{v}_{2}, t\right)
\end{array}
$$

Implementing a mean-field approximation which is valid in the proper thermodynamic limit $N \gg 1$ with $\beta N u \sim 1[48]$ :

$$
P_{2}\left(\mathbf{r}_{1}, \mathbf{v}_{1}, \mathbf{r}_{2}, \mathbf{v}_{2}, t\right)=P_{1}\left(\mathbf{r}_{1}, \mathbf{v}_{1}, t\right) P_{1}\left(\mathbf{r}_{2}, \mathbf{v}_{2}, t\right)+O(1 / N),
$$

we find that

$$
\frac{\partial P_{1}}{\partial t}+\mathbf{v}_{1} \frac{\partial P_{1}}{\partial \mathbf{r}_{1}}+\langle\mathbf{F}\rangle_{1} \frac{\partial P_{1}}{\partial \mathbf{v}_{1}}=\frac{\partial}{\partial \mathbf{v}_{1}}\left[D \frac{\partial P_{1}}{\partial \mathbf{v}_{1}}+\xi P_{1} \mathbf{v}_{1}\right]
$$

where

$$
\langle\mathbf{F}\rangle_{1}=-N \int d^{D} \mathbf{r}_{2} d^{D} \mathbf{v}_{2} \frac{\partial u}{\partial \mathbf{r}_{1}}\left(\mathbf{r}_{1}-\mathbf{r}_{2}\right) P_{1}\left(\mathbf{r}_{2}, \mathbf{v}_{2}, t\right) .
$$

This can be rewritten

$$
\frac{\partial f}{\partial t}+\mathbf{v} \frac{\partial f}{\partial \mathbf{r}}+\langle\mathbf{F}\rangle \frac{\partial f}{\partial \mathbf{v}}=\frac{\partial}{\partial \mathbf{v}}\left[D\left(\frac{\partial f}{\partial \mathbf{v}}+\beta f \mathbf{v}\right)\right]
$$

where

$$
\langle\mathbf{F}\rangle=-\nabla \Phi=-\int d^{D} \mathbf{r}^{\prime} \rho\left(\mathbf{r}^{\prime}, t\right) \frac{\partial u}{\partial \mathbf{r}}\left(\mathbf{r}-\mathbf{r}^{\prime}\right),
$$

is the mean-field force created by the particles.

\section{References}

[1] J. Binney and S. Tremaine, Galactic Dynamics, Princeton Series in Astrophysics, 1987.

[2] T. Padmanabhan, Phys. Rep. 188 (1990), 285.

[3] D. Lynden-Bell and R. Wood, Mon. Not. R. Astr. Soc. 138 (1968), 495.

[4] P. H. Chavanis, C. Rosier and C. Sire, Phys. Rev. E 66 (2002), 036105.

[5] P. H. Chavanis, Astron. Astrophys. 381 (2002), 340.

[6] P. H. Chavanis, Astron. Astrophys. 356 (2000), 1089. 
[7] J. D. Murray, Mathematical Biology, Springer, 1991.

[8] E. Keller and L. A. Segel, J. Theor. Biol. 26 (1970), 399.

[9] W. Jäger and S. Luckhaus, Trans. Amer. Math. Soc. 329 (1992), 819.

[10] V. A. Antonov, Vest. Leningr. Gos. Univ. 7 (1962), 135.

[11] H. Cohn, Astrophys. J. 242 (1980), 765.

[12] C. Lancellotti and M. Kiessling, Astrophys. J. 549 (2001), L93.

[13] H. Risken, The Fokker-Planck Equation, Springer, 1989.

[14] S. Chandrasekhar, Astrophys. J. 97 (1943), 255.

[15] P. H. Chavanis, Phys. Rev. E 68 (2003), 036108.

[16] P. H. Chavanis, P. Laurençot and M. Lemou, Physica A 341 (2004), 145.

[17] G. Wolansky, Nonlinear Anal. 24 (1995), 1119.

[18] P. H. Chavanis, J. Sommeria and R. Robert, Astrophys. J. 471 (1996), 385.

[19] C. Sire and P. H. Chavanis, Phys. Rev. E 66 (2002), 046133.

[20] P. H. Chavanis and C. Sire, Phys. Rev. E 69 (2004), 016116.

[21] C. Sire and P. H. Chavanis, Phys. Rev. E 69 (2004), 066109.

[22] P. H. Chavanis and C. Sire, Phys. Rev. E 70 (2004), 026115.

[23] M. Kiessling, J. Stat. Phys. 55 (1989), 203.

[24] C. Sire and P. H. Chavanis, Banach Center Publications, this volume.

[25] P. H. Chavanis, Phys. Rev. E 65 (2002), 056123.

[26] P. H. Chavanis, Phys. Rev. E 69 (2004), 066126.

[27] P. H. Chavanis, M. Ribot, C. Rosier and C. Sire, preprint.

[28] P. Biler, P. Laurençot and T. Nadzieja, Adv. Diff. Eq. 9 (2004), 563.

[29] P. H. Chavanis and M. Rieutord, A\&A 412 (2003), 1.

[30] P. H. Chavanis, Banach Center Publications, this volume.

[31] P. H. Chavanis, in preparation.

[32] P. H. Chavanis, Physica A 332 (2004), 89.

[33] A. Gamba, D. Ambrosi, A. Coniglio, A. de Candia, S. di Talia, E. Giraudo, G. Serini, L. Preziosi, F.A. Bussolino, Phys. Rev. Lett. 90 (2003), 118101.

[34] F. Filbet, P. Laurençot and B. Perthame, preprint.

[35] L. Onsager, Nuovo Cimento Supp. 6 (1949), 279.

[36] G. Joyce and D. Montgomery, J. Plasma Phys. 10 (1973), 107.

[37] P. H. Chavanis, in Dynamics and Thermodynamics of Systems with Long Range Interactions, T. Dauxois et al. (eds.), Lecture Notes in Physics, Springer, 2002 [condmat/0212223].

[38] P. H. Chavanis, Phys. Rev. E 64 (2001), 026309.

[39] P. H. Chavanis, Phys. Rev. E 58 (1998), R1199.

[40] R. Robert and J. Sommeria, Phys. Rev. Lett. 69 (1992), 2776.

[41] P. H. Chavanis, Phys. Rev. Lett. 84 (2000), 5512.

[42] F. Bouchet and T. Dumont, preprint [cond-mat/0305206].

[43] C. Rosier and L. Rosier, Applicable Analysis 75 (2000), 441.

[44] C. Rosier and L. Rosier, Quarterly of Applied Mathematics 61 (2003), 213.

[45] J. Pedlosky, Geophysical Fluid Dynamics, Springer, 1987.

[46] P. H. Chavanis, [cond-mat/0209098]

[47] F. Bouchet and J. Sommeria, J. Fluid Mech. 464 (2002), 165.

[48] P. H. Chavanis, preprint, cond-mat/0409641.

[49] N. Martzel and C. Aslangul, J. Phys. A 34 (2001), 11225.

[50] N. Martzel, PhD thesis, Université Paris 7 (2002). 